\title{
High-field calculations of Landau-like shallow donor states: A finite-difference approach
}

\author{
P. W. Barmby, J. L. Dunn, ${ }^{*}$ and C. A. Bates \\ Department of Physics, University of Nottingham, Nottingham, NG7 2RD, United Kingdom \\ T. O. Klaassen \\ Faculty of Applied Physics, Delft University of Technology, Delft Institute of Microelectronics and Submicrontechnology (DIMES), \\ P.O. Box 5046, 2600 GA Delft, The Netherlands \\ (Received 7 March 1997; revised manuscript received 1 December 1997)
}

\begin{abstract}
A theoretical method for calculating the energies and wave functions of an electron bound to a shallow donor in a semiconductor, subject to an applied magnetic field, is presented. This approach is particularly useful for describing highly excited Landau-like states, which cannot be dealt with properly using most other theoretical models. First, an adiabatic high-field approximation is used where mixing between different Landau-like states is neglected. Solving the one-electron Schrödinger equation is then reduced to finding solutions to a one-dimensional differential equation for motion along the field axis. We present results in which a finite-difference technique is used to solve this equation numerically. Values for the electron wave function at discrete points along the field axis are then determined. By calculating the discrete Fourier transform of this set of values, an analytical form for the wave function in terms of sines and cosines is obtained. These resultant wave functions are then used to calculate a Hamiltonian matrix in which mixing between different high-field states is included. Diagonalization of this matrix yields improved values for the energies of the impurity states. Where previous results exist, our results are compared with those of other theoretical approaches and from experiments on the donor in GaAs. The advantage of our approach is that it enables both energies and wave functions to be determined without any prior assumptions of the form of the wave function in the field direction. This includes expressions for highly excited states which are difficult to obtain by other means. The results demonstrate the validity of the present method of calculation. [S0163-1829(98)08315-5]
\end{abstract}

\section{INTRODUCTION}

The theoretical description of shallow donor impurities in semiconductor structures has been a major area of interest for many years. An exact analytical solution of the Schrödinger equation for the donor electron in a magnetic field has not yet been found and so approximate methods must be used. Many different approaches have been employed in both bulk and quantum-well (QW) systems, of which the most common have been perturbation, variational, numerical, and adiabatic methods. A good review of the earlier papers on bulk systems has been made by Zawadski. ${ }^{1}$ In addition, many recent references to work on multi-QW systems are given in Ref. 2.

In general, the influence of the semiconductor host on the interaction between a donor electron and the donor nucleus is taken into account through the introduction of an "effective" electron mass and the use of the proper dielectric constant of the medium. The problem is then equivalent to that of an isolated hydrogen atom in a magnetic field, except for field and energy scaling parameters. The effects of laboratory-strength magnetic fields can be equated to those of the intense magnetic fields that occur in the hydrogen-rich atmospheres of white dwarfs and neutron stars. Many calculations performed on hydrogen atoms in intense fields in order to understand the optical spectra obtained from these astrophysical objects can also be applied to the donor problem. A concise discussion of the differences between the hydrogen atom and a donor in a bulk semiconductor has been given by Klaassen. ${ }^{3}$
Solutions to both the donor problem (and hence the intense-field hydrogen problem) fall into two categories. First, because of the strong similarity with the hydrogen atom, a common approach for weak-field calculations is to treat the effect of the applied magnetic field starting from the basis of zero-field hydrogenlike states. ${ }^{4-10}$ However, many of the excited electron states observed experimentally in low-temperature bulk donor magneto-spectroscopy ${ }^{11-17}$ cannot be explained within this basis. An alternative view is to consider a strong-field picture in which the Coulomb interaction between the donor electron and the impurity ion is taken to be small. This allows the behavior of the electron perpendicular to the magnetic-field axis to be described by Landau wave functions. There is a direct correlation between some of the high-field Landau-like states and the low-field hydrogenlike states. ${ }^{17-21}$ However, there are in effect an infinite number of Landau-like states, generated by the highfield method, that do not have a hydrogenlike (zero-field) counterpart.

In this paper, we will refer to high-field states which correspond to hydrogenlike states as hydrogenic. The states can be labeled by three quantum numbers $n, l$, and $m$ by analogy to the hydrogen atom. High-field states which do not correspond to states in the hydrogenlike picture will be referred to as Landau-like. In other papers, these states are also referred to as metastable or autoionizing states. The Landau-like states can be labeled by the quantities $(N, m, \nu)$, where $N$ is the principal Landau index. $\nu$ refers to the number of nodes of the wave function in the field direction, and is introduced to distinguish between states with the same $N$ and $m$. 
It is obviously necessary to adopt a high-field approach if Landau-like states are to be described. One such approach will be adopted in this paper. As the motion of the electron perpendicular to the magnetic-field direction is described by Landau wave functions in these approaches, the calculation reduces to solving a one-dimensional problem for motion along the field direction. This equation can be solved approximately by a variety of variational and related methods, ${ }^{4,5,13,23-26}$ or by numerical approaches. ${ }^{19,22,26-29} \mathrm{~A}$ discussion of different variational wave functions was given in Ref. 30. Within manifolds of states with a given symmetry and magnetic quantum number $m$, the Landau-like states are nearly always excited states. As a result, the majority of the theoretical methods do not, or only with difficulty, yield reliable data on energies and wave functions of these states. Therefore, despite the large amount of work already performed, there is still a continuing interest in formulating a good model for these highly excited states in bulk III-V semiconductors. $^{11,12,31,32}$

The model will be developed for shallow donor impurities in bulk semiconductors, and applied particularly to GaAs in order to compare with experimental results. The results will also be compared to those from other theoretical models. By simple scaling of the magnetic field and energy, the results are directly applicable to donors in other semiconductors and to the hydrogen atom as well. Moreover, the method of calculation employed can be extended easily to describe impurities in QWs by modifying the wave functions to take the confinement effects of the QW potential into account. The QW case will not be considered here, because it is important that an accurate model for an impurity in the bulk case is formulated first of all. However, the viability of the method for QW and multi-QW devices will be discussed at the end of the paper in the context of future studies.

\section{THE THEORETICAL MODEL}

\section{A. The Hamiltonian of the system}

The Hamiltonian for a shallow donor impurity subject to a magnetic field $\boldsymbol{B}$ along the $z$ axis, can be written in the form

$$
\mathcal{H}=\mathcal{H}_{\rho \phi}+\mathcal{H}_{z}+\mathcal{H}_{r}
$$

where

$$
\begin{gathered}
\mathcal{H}_{\rho \phi}=-\frac{1}{\rho} \frac{\partial}{\partial \rho}\left(\rho \frac{\partial}{\partial \rho}\right)-\frac{1}{\rho^{2}} \frac{\partial^{2}}{\partial \phi^{2}}+\gamma L_{z}+\frac{\gamma^{2} \rho^{2}}{4} \\
\mathcal{H}_{z}=-\frac{\partial^{2}}{\partial z^{2}} \\
\mathcal{H}_{r}=-\frac{2}{\left(\rho^{2}+z^{2}\right)^{1 / 2}} .
\end{gathered}
$$

Due to the symmetry of this system, a symmetrical form for the magnetic vector potential has been used and the Hamiltonian expressed in terms of cylindrical coordinates. For the sake of generality, the Hamiltonian is written in dimensionless form such that lengths are given in units of effective
Bohr radii $a_{0}^{*}$ and energies are given in units of effective Rydbergs $R_{y}^{*}$. These two quantities are defined by the expressions

$$
a_{0}^{*}=\frac{\hbar^{2} 4 \pi \epsilon}{m^{*} e^{2}} \quad \text { and } \quad R_{y}^{*}=\frac{e^{2}}{8 \pi \epsilon a_{0}^{*}},
$$

where $\epsilon$ is the effective permittivity and $m^{*}$ is the effective mass of an electron in the medium under consideration. $L_{z}$ is the operator associated with the orbital angular momentum of an electron about the field axis, and $\gamma$ is a dimensionless form of the magnitude of the applied field given by

$$
\gamma=\frac{(4 \pi \epsilon)^{2} \hbar^{3}}{m^{* 2} e^{3}} B
$$

\section{B. High-field approximation}

If the effect of the Coulomb interaction on the donor electron is much less than that of the magnetic field in the direction perpendicular to the field axis, the component of the Hamiltonian operating on the $\rho$ and $\phi$ coordinates can be approximated to $\mathcal{H}_{\rho \phi}$ only. Also, the Coulomb interaction does not mix together different Landau states. The solution of the modified Schrödinger equation in the directions perpendicular to the field axis is then given by normalized Landau wave functions ${ }^{33}$

$$
\Phi_{N m}(\rho, \phi)=\left(\frac{\gamma}{2 \pi}\right)^{1 / 2}\left[\frac{n_{\rho} !}{\left(n_{\rho}+|m|\right) !}\right]^{1 / 2} e^{\xi / 2} \xi^{|m| / 2} L_{n_{\rho}}^{|m|}(\xi) e^{i m \phi},
$$

where $\xi$ is the dimensionless variable $\gamma^{2} \rho^{2} / 2$ and $n_{\rho}$ is the non-negative integer $N-(m-|m|) / 2$. Substituting the Landau wave function into Eq. (2.1), multiplying by its complex conjugate and integrating over the $\rho$ and $\phi$ directions leads to

$$
\mathcal{H}=2 \gamma\left(N+\frac{1}{2}\right)-\frac{\partial^{2}}{\partial z^{2}}+V_{N m}(z)
$$

where

$$
V_{N m}(z)=-2 \gamma^{1 / 2}\left[\frac{n_{\rho} !}{\left(n_{\rho}+|m|\right) !}\right]^{1 / 2} \int_{0}^{\infty} \frac{e^{-\xi} \xi^{|m|}\left[L_{n_{\rho}}^{|m|}(\xi)\right]^{2}}{\left(2 \xi+\gamma z^{2}\right)^{1 / 2}} d \xi .
$$

The Schrödinger equation then reduces to

$$
\mathcal{H} f^{N m}(z)=E f^{N m}(z),
$$

which is a one-dimensional differential equation in $z$. The function $f^{N m}(z)$ is the (as yet unknown) impurity wave function along the magnetic-field axis for given $N$ and $m$.

\section{Finite-difference calculation and Fourier transforms}

Equation (2.6) can be solved approximately using a number of different methods. Using a variational approach, which assumes a specific form for $f^{N m}(z)$, the values of $E$ for the impurity states can be estimated readily. ${ }^{4,5,23-26}$ The advantage of these methods is that $f^{N m}(z)$ obviously has an analytical form, which is useful when using the wave functions in further calculations such as of the polaron effect. However, as the form is assumed, this is likely to lead to 
inaccuracies in other aspects of the calculations. Another approach is to solve the equation using numerical methods. This is likely to give more accurate values for the energies than the variational approach but the drawback is that an analytical form for the wave function is not directly obtained. ${ }^{18,21,26-29}$

In this paper, we adopt a different approach to the impurity problem in which numerical results obtained using finite-difference techniques are Fourier transformed to obtain an approximate analytical form for the wave function. No prior assumption of the form of the wave function is made. The analytical wave functions are then used as basis functions for an improved calculation in which the limit of no admixing of Landau states imposed by the high-field limit is lifted. They are also used to obtain transition probabilities. The states are suitable for use as a basis for a calculation of the magnetopolaron effect, although this is not attempted in this paper.

The finite-difference approximation (as described in Ref. 34 , for example) is used to solve the one-dimensional differential equation (2.6) by dividing the $z$ direction into $n$ discrete points. A set of $n$ linear equations involving the values of $f^{N m}(z)$ at each point are deduced. These linear equations can be solved using matrix methods to obtain a series of $n$ possible values of $E$ and a corresponding set of $n$ values of $f^{N m}(z)$. The finite-difference approach requires knowledge of the boundary conditions for the differential equation. Therefore, for the case of an impurity in a bulk semiconductor material, we assume a finite width $L$ in the $z$ direction into which the donor electron is confined. The boundary conditions are therefore taken to be $f^{N m}(z)=0$ at $z= \pm L / 2$, taking the impurity to be at $z=0$. This confined case is expected to be equivalent to the bulk case.

Like other numerical methods, the finite-difference calculation described so far only produces discrete numerical values for $f^{N m}(z)$. However, these values can be used to obtain an approximate analytical form for the wave function by taking the discrete Fourier transform of the set of $n$ values $f^{N m}(z)$. This in turn allows the wave functions to be written as a combination of sine and cosine functions. The analytical forms for the $z$ component of the impurity wave function are thus

$f_{\nu}^{N m}(z)=\left(\frac{2}{L}\right)^{1 / 2} \sum_{k=1}^{n} \alpha_{k}^{N m \nu} \cos \left(\frac{k \pi}{L} z\right)$

for even-parity states,

$f_{\nu}^{N m}(z)=\left(\frac{2}{L}\right)^{1 / 2} \sum_{k=1}^{n} \alpha_{k}^{N m \nu} \sin \left(\frac{k \pi}{L} z\right)$

for odd-parity states, (2.7)

where $\alpha_{k}^{N m \nu}$ are the Fourier coefficients obtained from the transform of the $n$ values of the normalized functions $f_{\nu}^{N m}(z)$. The quantum number $\nu$ [corresponding to the number of nodes of $f^{N m}(z)$ along the $z$ axis] distinguishes between possible states with the same values for the quantum numbers $N$ and $m$.

\section{Admixing of Landau states}

The method discussed above employed the high-field approximation, in which the mixing between different Landau states by the Coulomb interaction was neglected. However, as analytical forms for the impurity wave function have been obtained in this limit, improvements can be made by using the states as basis functions for a more complete impurity wave function. Thus a basis state with quantum numbers $N$, $m$, and $\nu$ will have the form

$$
\psi_{N m \nu}=\Phi_{N m}(\rho, \phi) f_{\nu}^{N m}(z)
$$

As the Hamiltonian mixes Landau states having different values for $N$ and $\nu$, it is necessary to evaluate matrix elements of the form

$$
\begin{aligned}
\left\langle\psi_{N^{\prime} m \nu^{\prime}}|\mathcal{H}| \psi_{N m \nu}\right\rangle= & 2 \gamma\left(N+\frac{1}{2}\right) \delta_{N N^{\prime}} \delta_{\nu \nu^{\prime}} \\
& +\frac{\pi^{2}}{L^{2}} \sum_{k=1}^{n} \alpha_{k}^{N m \nu} \alpha_{k}^{N m \nu^{\prime}} k^{2} \times \delta_{N N^{\prime}} \\
& -2 \int_{-L / 2}^{L / 2} \int_{0}^{2 \pi} \int_{0}^{\infty} \frac{1}{\left(\rho^{2}+z^{2}\right)^{1 / 2}} \\
& \times \Phi_{N^{\prime} m}^{*}(\rho, \phi) \Phi_{N m}(\rho, \phi)\left[f_{\nu^{\prime}}^{N^{\prime} m}(z)\right] \\
& \times f_{\nu}^{N m}(z) \rho d \rho d \phi d z .
\end{aligned}
$$

If $N=N^{\prime}$, the results from the finite-difference calculation can be used directly. Also, since the diagonalization procedure in the finite-difference calculation results in orthogonal states, the matrix elements of the Hamiltonian could be replaced by the original value of the energy of a given state if $\nu=\nu^{\prime}$, or zero if $\nu \neq \nu^{\prime}$. However, these values for the energies are not used here for reasons which will be given in Sec. III. Therefore expression (2.9) is used to calculate all the matrix elements. A matrix is thus constructed, which ideally would encompass all values $\nu$ and an infinite set of values $N$. However, it is obviously necessary to restrict the matrix to a reasonable size by carrying out the summation to some maximum value $N_{\max }$. It is a straightforward matter to diagonalize the resulting matrix using computational means. The resulting impurity wave function is then written as

$$
\Psi=\sum_{N} \sum_{\nu} \beta_{N m \nu} \Phi_{N m}(\rho, \phi) f_{\nu}^{N m}(z)
$$

where the $\beta_{N m \nu}$ are coefficients obtained from the diagonalization procedure.

\section{Transition probabilities}

In order to ascertain which of the many impurity states predicted are most likely to be involved in experimental observations, the probability of transition from the ground state $[(0,0,0) \equiv 1 s]$ is calculated for each of the states using the impurity wave functions obtained in Eq. (2.10). In the Faraday configuration, where the transition-inducing radiation is applied in the same direction as the magnetic field, the transition probability is proportional to $\left|\left\langle\Psi^{\prime}|x| \Psi\right\rangle\right|^{2}$ (to a first approximation). For transitions to and from the ground state of the impurity, for which $m=0$, it can be shown that if $\Psi^{\prime}$ is an even parity state and $m^{\prime}=1$, then 


$$
\begin{aligned}
\left\langle\Psi^{\prime}|x| \Psi\right\rangle= & \sum_{N=0}^{N_{\max }-1} \sum_{\nu^{\prime}} \sum_{\nu} \sum_{k}\left(\frac{N+1}{2 \gamma}\right)^{1 / 2} \\
& \times \beta_{N+1 m^{\prime} \nu^{\prime}} \alpha_{k}^{N+1 m^{\prime} \nu^{\prime}}\left(\beta_{N m \nu} \alpha_{k}^{N m \nu}\right. \\
& \left.-\beta_{N+1 m \nu} \alpha_{k}^{N+1 m \nu}\right) .
\end{aligned}
$$

If $\Psi^{\prime}$ is an even-parity state and $m^{\prime}=-1$, then

$$
\begin{aligned}
\left\langle\Psi^{\prime}|x| \Psi\right\rangle= & \sum_{N=0}^{N_{\max }} \sum_{\nu^{\prime}} \sum_{\nu} \sum_{k}\left(\frac{N+1}{2 \gamma}\right)^{1 / 2} \\
& \times \beta_{N m \nu} \beta_{N m^{\prime} \nu^{\prime}} \alpha_{k}^{N m \nu} \alpha_{k}^{N m^{\prime} \nu^{\prime}} \\
& -\sum_{N=1}^{N_{\max }} \sum_{\nu^{\prime}} \sum_{\nu} \sum_{k}\left(\frac{N}{2 \gamma}\right)^{1 / 2} \\
& \times \beta_{N m \nu} \beta_{N-1 m^{\prime} \nu^{\prime}} \alpha_{k}^{N m \nu} \alpha_{k}^{N-1 m^{\prime} \nu^{\prime}} .
\end{aligned}
$$

The transition probability is otherwise zero in this configuration. In the Voigt configuration, for which the radiation is applied perpendicular to the magnetic field, the transition probability is proportional to $\left|\left\langle\Psi^{\prime}|z| \Psi\right\rangle\right|^{2}$. Therefore, the transition probability is nonzero if $\Psi^{\prime}$ is an odd-parity state and $m^{\prime}=0$ such that

$$
\begin{aligned}
\left\langle\Psi^{\prime}|z| \Psi\right\rangle= & \frac{2 L}{\pi^{2}} \sum_{N=0}^{N_{\max }} \sum_{\nu^{\prime}} \sum_{\nu} \sum_{k^{\prime}} \sum_{k} \beta_{N m \nu} \beta_{N m^{\prime} \nu^{\prime}} \alpha_{k}^{N m \nu} \\
& \times \alpha_{k}^{N m^{\prime} \nu^{\prime}}\left[\frac{1}{\left(k^{\prime}+k\right)^{2}}-\frac{1}{\left(k^{\prime}-k\right)^{2}}\right]
\end{aligned}
$$

The relative magnitudes of the transition probabilities involving each impurity state can be obtained directly from these equations. From these results, the states which are most likely to be involved in transitions to and from the ground state can be obtained.

\section{RESULTS AND DISCUSSION}

\section{A. Validation of the method}

The adiabatic finite-difference calculation described in Sec. II has been carried out initially for impurity states with quantum numbers $N=0$ and $m=0$ in a magnetic field for which $\gamma=1$. Although the model is not designed to work best for these predominantly low-field hydrogenlike states, we can conclude that if it works reasonably well for these states, it will work much better for the high-field Landau-like states. The width of the sample $L$ in the calculation was taken to be $100 a_{0}^{*}$ and the number of points used was chosen to be $n=199$. The results show that the ground state has even parity with no nodes $(\nu=0)$ along the field axis, and the next-lowest state is an odd-parity state with $\nu=1$. These states are therefore labeled $(0,0,0)$ and $(0,0,1)$, respectively. It is well known that these states correspond to the hydrogenlike $1 s$ and $2 p_{0}$ states, respectively. ${ }^{1}$ As the wave function $f(z)$ has already reduced to zero by $\left|z / a_{0}\right| \geqslant 10$, it seems reasonable to assume that these states will be unaffected by the finite width imposed on the bulk sample. In fact, this finite width only becomes important for highly excited impurity states. Since the confinement is in the $z$ direction only
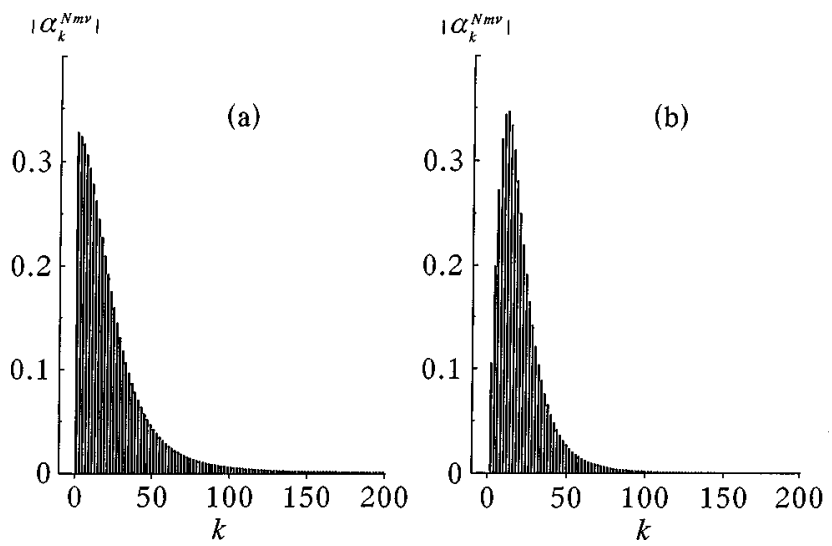

FIG. 1. A histogram of the magnitude of the Fourier coefficients as a function of the wave number $k$ for the two lowest $N=0, m$ $=0$ states.

and the quantum numbers $N$ and $m$ denote the behavior of the donor electron perpendicular to this direction, it also follows that only states with large $\nu$ will be affected.

The discrete Fourier transforms of the values of $f(z)$ for each of the low-lying states have been calculated to obtain the Fourier coefficients $\alpha_{k}^{N m \nu}$. The magnitudes of the coefficients with wave number $k$ for the $(0,0,0)$ and $(0,0,1)$ states are plotted as histograms in Figs. 1(a) and 1(b) respectively. It is found that for even-parity states, the values obtained for the Fourier coefficients for which $k$ is even are effectively zero. This is to be expected since we are imposing the condition that $f(z)=0$ at $z= \pm L / 2$; this is only satisfied for even-parity states if $k$ is odd. Similarly, the coefficients for odd-parity states are effectively zero if $k$ is even. Therefore, only half of the possible value of $k$ need to be considered for any given wave function. However, it should be noted that that if the impurity is positioned away from the center of the sample (or well), then odd- and even-parity states will mix and the wave functions will be composed of both sine and cosine terms. However, since we are looking at the problem of an impurity in a bulk material, it is reasonable to impose artificial boundary conditions which are symmetric about the impurity center.

As can be seen in Fig. 1, the magnitudes of the Fourier coefficients fall off almost to zero with larger values of $k$. Therefore, we expect the accuracy of the results to change only slightly if the number of points $n$ in the finite-difference calculation, and therefore the number of sine/cosine terms in the wave function $f(z)$, is increased above $n=199$. We also expect that for states with higher values of $\nu$, the higher $k$ terms will be increasingly less important. This is because these states are energetically more excited and therefore not as strongly bound to the impurity ion by the Coulomb interaction. This will result in less mixing of free-electron states. This conclusion is supported by the histogram shown in Fig. 1 for the $(0,0,1)$ state which shows a fall off slightly quicker with increasing $k$ than that found with the $(0,0,0)$ state.

The effect on the energy of the $(0,0,0)$ ground state of varying $n$ up to 199 is shown in Fig. 2. The figure shows both the energies obtained directly from the finite-difference calculation and the energies obtained using the wave functions from the Fourier transformation to calculate the Hamiltonian matrix followed by its diagonalization to find the im- 


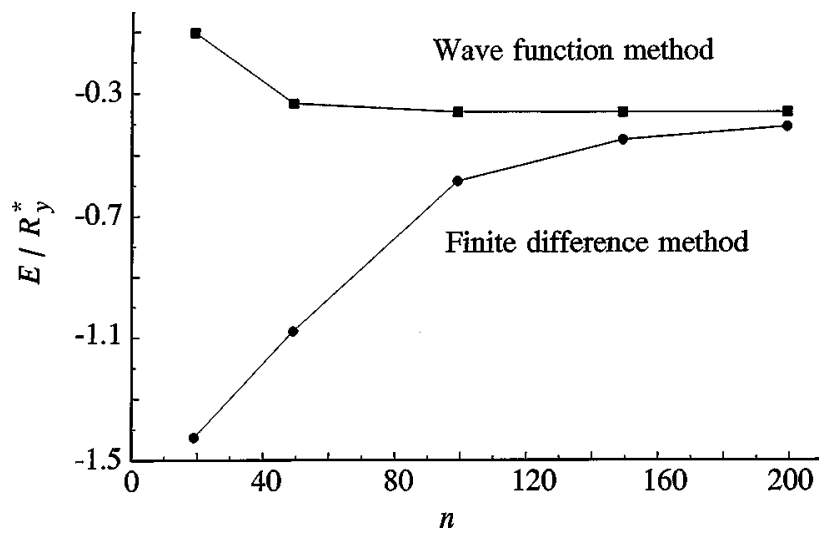

FIG. 2. Variation of the ground-state energy with the number of points $n$ used as calculated by the finite-difference method $(\boldsymbol{O})$ and by the wave-function method ( $\mathbf{\square})$.

purity energies. For simplicity, the Hamiltonian matrix was constructed using $N=0$ states only. As can be seen, both methods appear to be converging to some definite value for the ground-state energy as $n$ increases. However, the wavefunction method appears to approach this value more quickly than the finite-difference method, and to have a smaller dependence on $n$. This is why, when the Hamiltonian matrix elements are calculated as described in Sec. II C 1, we do not use the energy value directly from the finite-difference method alone. We feel that the wave function result has converged sufficiently by $n=199$ to render further computationally intensive calculations with higher values of $n$ unnecessary.

\section{B. Ionization energies}

As the method presented here is essentially a high-field approach it can be expected to work best for the Landau-like states. They are also the states which have received least attention in the literature. We will therefore concentrate on these now. Figure 3 shows a plot of the ionization energies of the $(1,0,1),(1,-1,0),(3,1,0)$, and $(4,1,0)$ metastable states calculated using our method. (The scatter on our points is due to taking only $n=199$ points in the calculations.) The

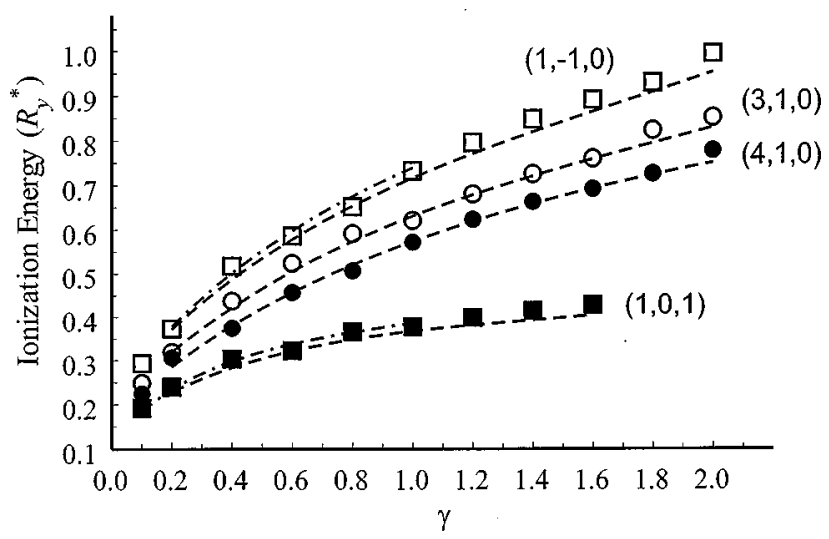

FIG. 3. A plot of ionization energy against magnetic field for the Landau-like states $(1,0,1),(1,-1,0),(3,1,0)$, and $(4,1,0)$. The circles and squares are the results of the current calculations. The dashed lines are the variational results of Barmby et al. (Ref. 11) and the dot-dashed lines the results of Friedrich and Chu (Ref. 13). figure also reproduces results of the variational method of Barmby et al. ${ }^{1}$ It can be seen that the agreement between the two approaches is excellent for the $(3,1,0)$ and $(4,1,0)$ states. The ionization energies predicted here for the $(1,0,1)$ and $(1,-1,0)$ states are higher than the variational results: a plot on an expanded scale shows the differences to be around $0.03 R_{y}^{*}$. However, this means the variational state has a higher actual energy than that predicted here, so our new state is a better approximation to the true state than the variational one. Results obtained up to $\gamma=1$ for the $(1,0,1)$ and $(1,-1,0)$ states by Friedrich and $\mathrm{Chu}^{22}$ using a standard discretization technique have also been plotted on the figure. They agree very closely with those of the current calculations.

Experimental magneto-optical data has already been obtained on impurities in bulk GaAs. ${ }^{11}$ The experimental data has been converted to dimensionless units and stripped of its material-specific character by removing the small band nonparabolicity and nonresonant electron-phonon coupling effects. A correction was also applied to account for the change in effective mass with field and energy. These results were then found to agree well with the results of the variational calculations. ${ }^{11}$ The variational calculations have recently been repeated and extended to higher excited states and material-specific effects included in the parametrization. ${ }^{18}$ These authors also find excellent agreement between these results and experiment.

\section{Hydrogenic states}

Although our method is not expected to be as accurate for low-lying hydrogenic states as for the Landau-like states, it is instructive to compare our results with those of the many previous calculations for these states. ${ }^{4-10}$ Here, we will concentrate on the results of Makado and $\mathrm{McGill}^{8}$ because they give tables of values for fields up to $\gamma=5$. However, the choice is not important as the discrepancies between Makado and McGill's results and those of others is much smaller than the experimental uncertainties on magneto-optical results for impurities in GaAs. For example, the differences between their predictions for the $1 s$ and $2 s$ states and those of Praddaude ${ }^{9}$ or Rösner et al. ${ }^{27}$ are at most $2.8 \times 10^{-4} R_{y}^{*}$, and with those of Shi et al. ${ }^{10} 0.018 R_{y}^{*}$.

Figure 4 shows the calculated energies for the $(0,0,0)$, $(0,0,2)$, and $(1,1,0)$ states, which correspond to hydrogenic $1 s, 2 s$, and $2 p_{+1}$ states, respectively, together with the results of Ref. 8. The results are given as a function of magnetic field both without admixing of different Landau states and with admixing up to $N_{\max }=7$. It can be seen that there is a significant discrepancy between the energy of the $(0,0,0)$ ground state excluding admixing and the result of Makado and McGill. ${ }^{8}$ This is because the donor electron in its ground state is tightly bound to the impurity ion by the Coulomb interaction and so a theoretical method (such as ours) which does not take into account the effect of the Coulomb interaction is unlikely to be accurate. However, when the admixing of Landau states is included, there is much better agreement for higher values of field. Nevertheless, the method described here is still inaccurate at the lower values of $\gamma$. Although possibly the problem at these lower $\gamma$ values could be reduced by taking a larger value of $N_{\max }$ the construction 


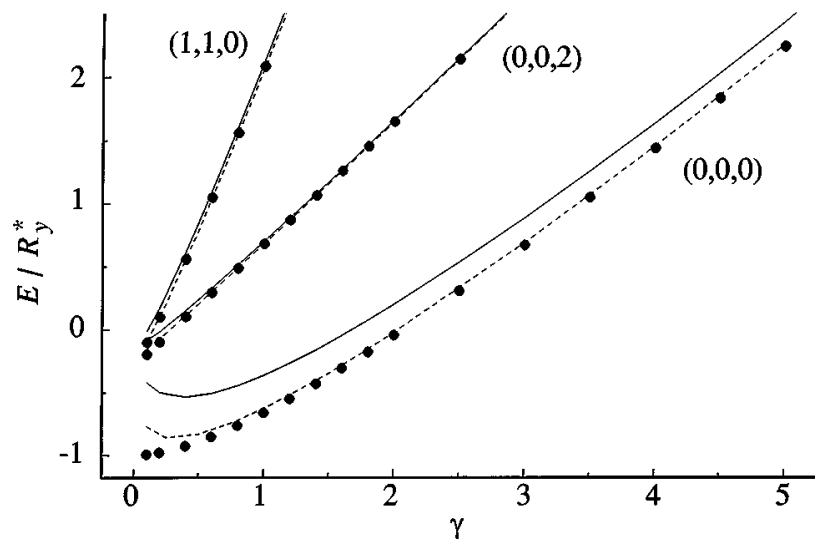

FIG. 4. Comparison of the energies calculated by Makado and McGill (Ref. 12) (O) with those derived here for the hydrogenic states $(0,0,0),(0,0,2)$, and $(1,1,0)$. Both sets of results are plotted without admixing of other states (solid line) and with admixing included (dashed line).

of a wave function in terms of Landau states in this regime remains inappropriate for a situation where the effect of the magnetic field is negligible compared to that of the Coulomb interaction.

Although the energies of the $(0,0,2)$ and $(1,1,0)$ impurity states neglecting the admixing of Landau levels deviate significantly from the hydrogenic values, the values including admixing are still close to those of hydrogenic methods. At $\gamma=0.1$, the difference between the energies obtained with admixing included and those from the variational calculations are $0.04 R_{y}^{*}$ and $0.01 R_{y}^{*}$ for the $(0,0,2)$ and $(1,1,0)$ states, respectively. For these excited states, the donor electron is not as tightly bound to the impurity ion as in the case of the ground state and so the Coulomb interaction is not so dominant. Hence the high-field approach is a more reasonable approximation. It can be seen from the figure that the agreement between the results of the present approach and those of weak-field models becomes even better towards higher magnetic field.

\section{Transition energies}

The energies calculated above can also be used to predict transition energies. These results can then be compared to experimental transition energies obtained for impurities in $n$-type GaAs. ${ }^{11,12}$ As our calculations are not accurate for the ground state, we have calculated our energies relative to the energy of the $(0,0,0)$ state obtained by Makado and McGill. ${ }^{8}$ To express the calculated values for magnetic fields and energies in nondimensionless units appropriate to GaAs, values of $\epsilon=12.5 \epsilon_{0}, \quad m^{*}=0.067 m_{e}, \quad a_{0}^{*}=98.7 \AA, \quad$ and $R_{y}^{*}$ $=5.83 \mathrm{meV}=47.0 \mathrm{~cm}^{-1}$ were used.

When admixing is included, a whole set of impurity energies is produced; it is not immediately obvious which states should be considered. Therefore we have examined the magnitudes of the wave-function coefficients $\beta_{N m \nu}$ from Eq. (2.10) to find the dominant basis state involved in a given admixed state. In this way, a given admixed state can be labeled $(N, m, \nu)$ according to its dominant contribution. To confirm this identification, the transition probability between each selected state and the ground state has been calculated

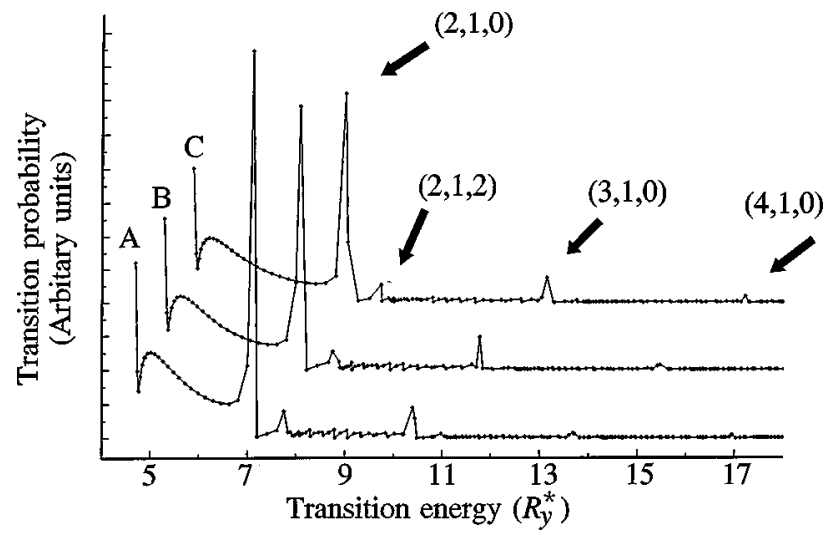

FIG. 5. A plot of the transition probabilities for the $m=1$ states as a function of their calculated transition energies. Three different plots are given for different values of $\gamma: A=1.6, B=1.8$, and $C$ $=2.0$.

using Eqs. (2.11), (2.12), and (2.13). A plot of the transition probabilities for $m=1$ states in the Faraday configuration against their theoretical transition energies for different $\gamma$ values is given in Fig. 5. The $(1,1,0)$ and $(1,1,2)$ states were omitted in order to highlight the peaks belonging to more excited Landau-like states, as their transition probabilities are much greater than those shown. The results for a given field have also been joined by a solid line as a guide to the eye. For the calculation of these transition probabilities, the present method was used to estimate the form for the ground-state wave function although the ground-state energy values of Ref. 8 were used to calculate the transition energies. The figure shows clear transition peaks, each varying in position with different $\gamma$ values.

The theoretical and experimental results for transitions from the $(0,0,0)$ ground state have been plotted for a range of magnetic fields and impurity states in Figs. 6(a)-6(d). Many of these excited states are difficult to predict using other models. These figures show that the theoretical transition energies agree very well with the experimental values. They are most accurate for high-energy states, although the calculations do tend to consistently overestimate the transition energies at higher fields. This deviation is probably the result of not correcting for material-specific factors, of which the most important will be the polaron interaction involving the donor electron and longitudinal-optical phonons. This is known to be very important for fields at which anticrossing of impurity transition energies occurs in the region of $300 \mathrm{~cm}^{-1}$. However, away from this region, calculations ${ }^{35}$ indicate that the effect induces an energy lowering of the order of $1.5 \mathrm{~cm}^{-1}$. As calculations of the polaron effect involve using secondorder perturbation theory and the Frölich Hamiltonian, ${ }^{35}$ it is necessary to have an analytical form for the impurity wave functions. Hence the results of our calculations could be used directly here. Indeed, since the impurity wave function is calculated in terms of sine and cosine functions, such a calculation will be simpler with this method than with other methods. This is an area for future consideration, especially if the procedure is extended to quantum wells where it is known that the polaron effect is measurably larger. ${ }^{36}$

\section{CONCLUSIONS}

In this paper, we have put forward a theoretical approach to modeling the properties of shallow donor impurities in 

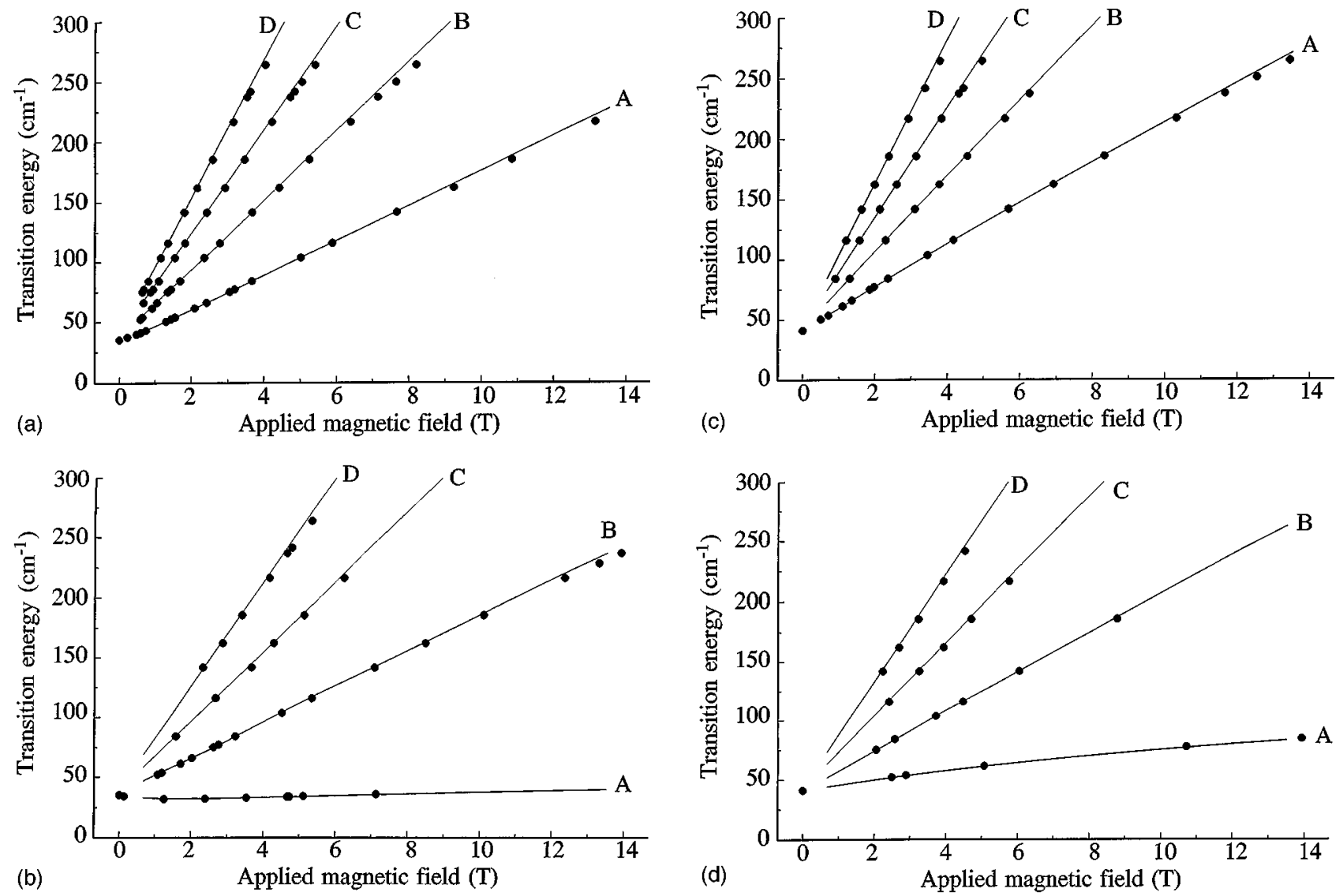

FIG. 6. A comparison of calculated transition energies (solid lines) and the corresponding experimental values (O) for transitions from the $(0,0,0)$ ground state to various states $(N, m, \nu)$ : (a) $A=(1,1,0), B=(2,1,0), C=(3,1,0)$, and $D=(4,1,0),($ b) $A=(0,-1,0), B=(1$, $-1,0), C=(2,-1,0)$, and $D=(3,-1,0),(\mathrm{c}) A=(1,1,2), B=(2,1,2), C=(3,1,2)$, and $D=(4,1,2),(\mathrm{d}) A=(0,-1,2), B=(1,0,1), C$ $=(2,0,1)$, and $D=(3,0,1)$.

bulk semiconductors. In particular, the highly excited Landau-like states can be dealt with accurately. The method has the advantage of not assuming a form for the wave functions of the impurity states, while at the same time being able to obtain analytical expressions for these wave functions. Furthermore, impurity wave functions are expressed in the relatively simple form of a linear combination of sine and cosine functions which will make further calculations easier; for example, the calculation of transition probabilities or the possible estimation of the polaron correction. Comparing the theoretical results from this approach with those from other models, both for hydrogenlike and Landau-like states, and available experimental data for bulk $n-\mathrm{GaAs}$, the method has been shown to predict accurately the energies of excited impurity states very well.

The method used for the finite-difference calculation modeled a bulk sample by a sample of large finite width $L$ along the magnetic-field direction. The model is in fact that of a very wide quantum well. By reducing the value of $L$, the theoretical approach can therefore be applied to the modeling of impurities in QW structures. It should also be a relatively simple matter to modify the theory to describe impurities in MQW structures. Because the method does not require an assumed form for the impurity wave function, the effect of the varying barrier potential can simply be taken into account in the Hamiltonian. For magnetic fields applied perpendicularly to the barriers, only $f^{N m}(z)$ needs to be modified, and this simply affects the equation to be solved by the finitedifference method.

\section{ACKNOWLEDGMENTS}

The authors would like to thank Dr. Mark Fromhold and Paul Wilkinson for their help in understanding the finitedifference method.
*Author to whom correspondence should be addressed.

${ }^{1}$ W. Zawadzki, Landau Level Spectroscopy, edited by G. Landwehr and E. I. Rashba (Elsevier, Amsterdam, 1991).

${ }^{2} 23$ rd International Conference on the Physics of Semiconductors, edited by M. Scheffler and R. Zimmermann (World Scientific, Singapore, 1996).

${ }^{3}$ T. O. Klaassen, C. R. Acad. Sci. Paris, Ser. IIb 323, 187 (1996).
${ }^{4}$ Y. Yafet, R. W. Keyes, and E. N. Adams, J. Phys. Chem. Solids 1, 137 (1956).

${ }^{5}$ R. F. Wallis and H. J. Bowlden, J. Phys. Chem. Solids 7, 78 (1958).

${ }^{6}$ C. Aldrich and R. L. Greene, Phys. Status Solidi B 93, 343 (1979).

${ }^{7}$ R. L. Greene and K. K. Bajaj, Solid State Commun. 45, 825 (1983). 
${ }^{8}$ P. C. Makado and N. C. McGill, J. Phys. C 19, 873 (1986).

${ }^{9}$ H. C. Praddaude, Phys. Rev. A 6, 1321 (1972).

${ }^{10}$ J. M. Shi, F. M. Peeters, and J. T. Devreese, Phys. Rev. B 48, 5202 (1993).

${ }^{11}$ P. W. Barmby, J. L. Dunn, C. A. Bates, and T. O. Klaassen, Phys. Rev. B 54, 8566 (1996).

${ }^{12}$ P. W. Barmby, J. L. Dunn, C. A. Bates, E. P. Pearl, C. T. Foxon, A. J. van der Sluijs, K. K. Geerinck, T. O. Klaassen, A. van Klarenbosch, and C. J. G. M. Langerak, J. Phys.: Condens. Matter 6, 7867 (1994).

${ }^{13}$ H. P. Wagner and W. Prettl, Solid State Commun. 66, 367 (1988).

${ }^{14}$ A. J. van der Sluijs, K. K. Geerinck, T. O. Klaassen, and W. Th. Wenckebach, Proc. SPIE 2104, 513 (1993).

${ }^{15}$ A. van Klarenbosch, K. K. Geerinck, T. O. Klaassen, W. Th. Wenckebach, and C. T. Foxon, Europhys. Lett. 13, 237 (1990).

${ }^{16}$ Z. Chen, Z. Chen, P. L. Liu, G. L. Shi, C. M. Hu, X. H. Shi, and S. C. Shen, J. Appl. Phys. 81, 6183 (1997).

${ }^{17}$ A. van Klarenbosch, T. O. Klaassen, W. Th. Wenckebach, and C. T. Foxon, J. Appl. Phys. 67, 6323 (1990).

${ }^{18}$ D. Wintgen and H. Friedrich, J. Phys. B 19, 991 (1986).

${ }^{19}$ R. J. Elliott and R. Loudon, J. Phys. Chem. Solids 15, 196 (1960).

${ }^{20}$ W. S. Boyle and R. W. Howard, J. Phys. Chem. Solids 19, 181 (1961).

${ }^{21}$ J. Simola and J. Virtamo, J. Phys. B 11, 3309 (1978).
${ }^{22}$ H. Friedrich and M. Chu, Phys. Rev. A 28, 1423 (1983).

${ }^{23}$ A. G. Zhilich and B. S. Monozon, Sov. Phys. Solid State 8, 2846 (1967).

${ }^{24}$ V. G. Golubev, V. I. Ivanov-Omskii, A. V. Osutin, R. P. Seisyan, Al. L. Efros, and T. V. Yaseva, Sov. Phys. Semicond. 22, 896 (1988).

${ }^{25}$ H. Friedrich, Phys. Rev. A 26, 1827 (1982).

${ }^{26}$ V. Canuto and D. C. Kelly, Astrophys. Space Sci. 17, 277 (1972).

${ }^{27}$ W. Rösner, G. Wunner, H. Herold, and H. Ruder, J. Phys. B 17, 29 (1984).

${ }^{28}$ M-C. Chu and H. Friedrich, Phys. Rev. A 28, 3651 (1983).

${ }^{29}$ S. K. Bhattacharya and S-I. Chu, J. Phys. B 16, L471 (1983).

${ }^{30}$ S. Narita and M. Miyao, Solid State Commun. 9, 2161 (1971).

${ }^{31}$ Y-M. Ming Mu, J-P. Peng, P-L. Liu, S-C. Shen, and J-B. Zhu, Phys. Rev. B 48, 10864 (1993).

${ }^{32}$ W. Zawadzki, P. Pfeffer, S. P. Najda, H. Yokoi, S. Takeyama, and N. Miura, Phys. Rev. B 49, 1705 (1994).

${ }^{33}$ L. D. Landau and E. M. Lifshitz, Quantum Mechanics (Nonrelativistic Theory) 3rd ed. (Pergamon, New York, 1977).

${ }^{34}$ O. C. Zienkiewicz and K. Morgan, Finite Elements and Approximation (Wiley, New York, 1983).

${ }^{35}$ J-P. Cheng, B. D. McCombe, J. M. Shi, F. M. Peeters, and J. T. Devreese, Phys. Rev. B 48, 7910 (1993).

${ }^{36}$ F. M. Peeters and J. T. Devreese, Phys. Rev. B 31, 3689 (1985). 